\title{
A New Numerical Approach to the Calibration and Interpretation of PEA Measurements
}

\author{
A. A. Mulla, S. J. Dodd, N. M. Chalashkanov and L. A. Dissado \\ University of Leicester \\ University road \\ Leicester, LE1 7RH, UK
}

\begin{abstract}
In this paper, a new approach for interpreting raw PEA signals is proposed. The theory behind acoustic wave propagation in ideal (no attenuation and no dispersion) materials and in real materials (with attenuation and dispersion) initially free of space charge is described. A simulation model has been developed for the following: (1) the acoustic signal formation in the PEA apparatus, the transmission of the acoustic waves, their attenuation/dispersion and detection; (2) the instrumental effects of having a capacitive piezoelectric sensor driving a $50 \mathrm{Ohm}$ input impedance amplifier. The various layers of a PEA system were considered in the model and the effect of each layer on the acoustic wave propagation is analyzed. Since the model allowed raw PEA data to be simulated, it can be used to identify potential sources of error in interpreting real PEA measurement data, such as acoustic mismatch between PEA layers, electrode material effects, pulse voltage, etc. The results showed good agreement between the simulated and experimentally obtained data in the case of space charge free samples.
\end{abstract}

Index Terms - pulsed electroacoustic (PEA) measurements, finite differences method (FDM), acoustic propagation and attenuation

\section{INTRODUCTION}

THE pulsed electroacoustic (PEA) method has been widely used to detect space charge accumulation inside dielectric materials due to its simplicity and practicality [1]. The basic operational principles behind the PEA method are well understood. A typical PEA system consists of a top electrode, a ground electrode, a piezoelectric sensor (PVDF) and an amplifier. An externally applied pulsed electric field exerts a momentary mechanical force on space charge within the insulating material. The force exerted couples to the insulation material and hence produces acoustic pressure waves inside the dielectric in proportion to the space charge density [2]. These acoustic waves propagate through the sample and earthed aluminium (Al) bottom electrode and are detected and transformed into an electrical signal by a piezoelectric transducer/amplifier [3]. However, such a simplistic description hides a number of assumptions that have to be reconciled before reliable space charge measurements can be obtained. It is also known that various factors can affect the quality of the measured PEA signal such as acoustic reflections at material interfaces, attenuation and dispersion of the acoustic signal as it propagates through the sample material and the frequency response of the piezoelectric sensor/amplifier combination. The material properties, especially those of the test sample and the electrodes, have direct impact on the acoustic wave generation,

Manuscript received on 17 August 2019, in final form 13 November 2019, accepted xx Month 20yy. Corresponding author: S. J. Dodd. propagation and absorption, for example when there is a mismatch between the acoustic properties of the dielectric sample and the acoustic properties of the top electrode (usually Semicon) $[4,5]$. This is also true for the sensor side of the PEA layers, where there are differences in the properties of the PMMA and PVDF layers. Often, in the PEA measurement literature, the contribution of the pulse voltage is neglected which leads to the introduction of a significant error, especially when the ratio of the pulse voltage to the applied DC voltage is high [6]. Numerical techniques are often employed to recover the authentic signal related to the presence of space charge within the sample. The main approach adopted in the literature uses deconvolution algorithms [6]. This involves deconvolution of the various instrumental responses and introduction of corrections for the acoustic attenuation and dispersion [7]. Calibration of the system is also required. Although transfer functions can be used to describe the signal propagation, the correct estimation of the values of the coefficients of attenuation and dispersion from the raw PEA data presents a challenge [8]. In this paper, a numerical approach has been applied to the simulation of the whole PEA measurement process. This encapsulates the formation of the acoustic pressure waves, their propagation and detection. The aim is to provide a quantitative framework in order to assess the effect of the various PEA instrumental and sample factors that potentially modify the experimental PEA raw output data. Ultimately, the PEA simulation is intended as a tool that will allow raw PEA experimental data to be predicted from charge transport theory leading to better understanding of space charge accumulation in dielectrics. 


\section{ONE-Dimensional PEA MODEL 2.1 BACKGROUND THEORY}

When a pulsed electric field is applied across a dielectric slab, a force is exerted on the dielectric. The total force density (the force acting per unit volume of the dielectric) is described in $[9,10]$. In the present model the total force vector, which in the absence of a permanent dipole density, is given by:

$$
\vec{f}=\rho_{c} \cdot \vec{E}-\frac{\varepsilon_{0}}{2} \cdot E^{2} \cdot \vec{\nabla} \varepsilon_{r}-\frac{1}{2} \vec{\nabla}\left(a \cdot E^{2}\right)
$$

where $\vec{f}$ is the force density, $\vec{E}$ is the electric field, $\rho_{c}$ is the space charge density, $\varepsilon_{0}$ is the permittivity of vacuum, $\varepsilon_{r}$ is the relative permittivity of the dielectric and $a$ is the electrostriction coefficient of the dielectric. The first term on the right hand side represents the columbic force density due to the electric field, $E(x)$. The second term represents a force density due to nonuniformity of the permittivity of the dielectric. The final term is the dielectrophoretic force density; it is generated when the square of the electric field $\mathrm{E}$ has a spatial variance.

The calculation of the force density can be simplified by expressing $\vec{f}$ as the divergence of Maxwell's tensor $\mathrm{M}_{i j}$. The main advantage of this method of calculation is that the force vector can be written in terms of a vector electric field without requiring the calculation of the interfacial surface charge densities at each of the two electrodes of the PEA system. Therefore, by using Gauss's equation to eliminate the space charge density $\left(\rho_{c}\right)$, the force density vector can be written as follows [11]:

$$
\overrightarrow{f_{l}}=\frac{\partial M_{i j}}{\partial x_{j}}=\frac{\partial\left(\varepsilon E_{i} E_{j}\right)}{\partial x_{j}}-\frac{1}{2} \frac{\partial\left((\varepsilon+a) E^{2}\right)}{\partial x_{i}}
$$

and hence Maxwell's tensor is:

$$
M_{i j}=\varepsilon E_{i} E_{j}-\frac{1}{2}(\varepsilon+a) \delta_{i j} E^{2}
$$

where $\delta_{i j}$ is the Kronecker delta function having matrix elements of value, 1 when $i=j$ and zero otherwise. The PEA is a 1-D measurement system and in this case Maxwell's tensor becomes a scalar quantity:

$$
M=\frac{1}{2}(\varepsilon-a) E^{2}
$$

During the time of application of the pulse voltage, the field $E$ becomes $E+\Delta E$ and Maxwell's tensor becomes $M+\Delta M$. Assuming that the voltage pulse is of sufficiently short duration not to affect the existing space charge density [4], the change in Maxwell's tensor:

$$
\Delta M=(\varepsilon-a) E \Delta E+\frac{1}{2}(\varepsilon-a) \Delta E^{2}
$$

$\Delta \mathrm{M}$ has units of force per metre squared, and therefore can be interpreted as pressure in the dielectric exerted due to a transient change in the applied electric field $\Delta \mathrm{E}$. It can be seen above that the effect of electrostriction is to alter the source pressure from a factor $\varepsilon$ to $(\varepsilon-a)$. Here, we assume that the electrostriction coefficient is zero.

The time dependent applied voltage pulse, $g_{p}(t)$, that produces the field increment, $\Delta E$, is assumed to have a Gaussian wave shape and is given by:

$$
g_{p}(t)=e^{\frac{-4 \ln (2) \cdot\left(t-t_{p}\right)^{2}}{t_{w}}}
$$

where $t_{p}$ is the pulse delay time and $t_{w}$ is the pulse width at the $37 \%$ amplitude. Therefore, the time dependent pulsed electric field, $\Delta E(x, t)$, is:

$$
\Delta E(t)=E_{p} \cdot g_{p}(t)
$$

where, $E_{p}$ is the magnitude of the pulse field within the sample. In a thin film (1D geometry) of constant permittivity, $E_{p}$, is a constant, independent of position, and given by $\frac{\Delta V}{d}$, where $\Delta V$ is the magnitude of the pulsed voltage and $d$ is the sample thickness.

The pressure variation produced by the applied electric pulse field will induce longitudinal acoustic waves inside the dielectric. The propagation of longitudinal acoustic waves are governed by the wave equation:

$$
\frac{\rho \partial^{2} p}{\partial t^{2}}=\frac{\partial}{\partial x}\left(C \frac{\partial p}{\partial x}\right)+\text { sources }
$$

where, $p$, represents the pressure density and $C$ the effective modulus of the medium. The wave equation for longitudinal wave propagation in solid media can be re-expressed as two first order differential equations [12]:

$$
\begin{aligned}
& \frac{\partial p}{\partial t}=-C \frac{\partial v}{\partial x}+\frac{\partial M}{\partial t} \\
& \frac{\partial v}{\partial t}=-\frac{1}{\rho} \frac{\partial p}{\partial x}
\end{aligned}
$$

Here, the coordinate, $x$, is the principle coordinate along the sample thickness, $p$ is the pressure density, $v$ is the vector velocity in the $x$ direction, $C$ is the effective modulus $\rho$ is the mass density of the material. The source term for pressure waves from Maxwell's tensor is also included in equation (9). The effective modulus can be calculated from the product of the material density, $\rho$, and the square of the sound velocity, $c$ [12].

$$
C=\rho c^{2}
$$

\subsection{FINITE DIFFERENCE TIME DOMAIN METHOD}

The finite difference time domain (FDTD) method was used to simulate the acoustic wave propagation in 1D. Figure 1 shows the 1D computational domain used for the FDTD computations. The spatial domain was divided into subdomains corresponding to the 6 layers of the PEA system sandwiched between two perfectly matched layers (PMLs) [13] at each side of the domain as shown in Figure 1. The domain contains a finite number of equally spaced nodes of separation, $\Delta x$. The PML regions were not part of the PEA apparatus but are necessary in order to absorb pressure waves leaving the domain so that they do not reflect back into the domain.

The layers in Figure 1 represent: (1) top PML absorber, (2) top $\mathrm{Al}$ electrode of the PEA apparatus, (3) semicon layer, (4) test sample, (5) bottom Al electrode, (6) PVDF sensor, (7) PMMA, and (8) bottom PML absorber.

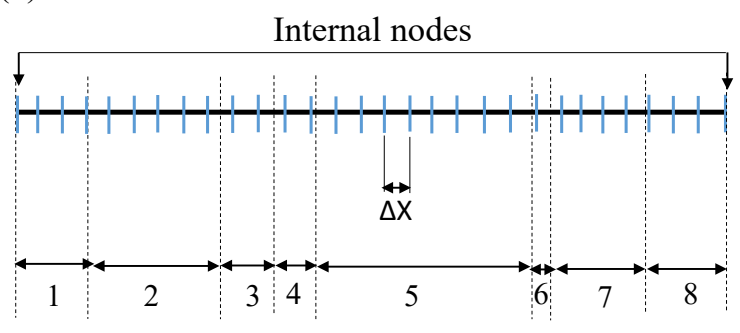

Figure 1. Discretized PEA layers based on FDM.

Equations (9) and (10) governing the wave propagation were discretized forming the following set of algebraic equations [8]: 


$$
p^{n+1}(i)=p^{n}(i)-\frac{1}{2} C(i) \frac{\Delta t}{\Delta x}\left(v^{n}(i+1)-v^{n}(i-1)\right)+\left(M(i)^{n+1}-M(i)^{n}\right)
$$

$v^{n+1}(i)=v^{n}(i)-\frac{1}{2 \rho(i)} \frac{\Delta t}{\Delta x}\left(p^{n}(i+1)-p^{n}(i-1)\right)$

where the superscript $n$ is the time iteration index, $\Delta x$ and $\Delta t$ are the spatial and time steps, respectively, $i$ refers to the node index in $x$ direction. For the PML layers, Equations (9), (10), (12) and (13) were modified to include a spatially quadratic absorption term as detailed in reference [13] to ensure no reflections at the two ends of the domain.

The PEA output voltage as a function of time, $V_{P V D F}(t)$, can then be obtained by integrating the pressure wave along the thickness of the PVDF sensor [14] at each time iteration $\Delta t$.

\subsection{MODELLING ACOUSTIC ATTENUATION AND DISPERSION IN PEA OUTPUT SIGNAL}

Here a method is proposed for the inclusion of attenuation and dispersion as pressure waves propagate through the dielectric. Although the propagation of acoustic waves through an absorbing and dispersive media could be taken into account by modification of the propagation equations (9) and (10), an easier method is to correct for attenuation and dispersion by post analysis of the simulated PEA signal. The aim is to carry out attenuation and dispersion correction on an idealized PEA response such that the instabilities associated with the traditional space charge recovery method [7] can be avoided. Therefore in this new method, the numerically unstable calculation of the inverse transfer function for the $G$ matrix $\left(G(t, z)^{-1}\right)$ will no longer be required. The attenuation and dispersion coefficients of an assumed Gaussian acoustic pressure pulse [15] were calculated in the following way.

The attenuation/dispersion correction is carried out based on equation (14) by considering a time dependent Gaussian pulse propagating in the $\mathrm{x}$ direction over a time step, $\Delta t$. After each time step, the pulse has propagated a distance $c \Delta t$ and is attenuated and dispersed by a specified amount defined by the change of the pulse $G(t)$ to $G(t+\Delta t)$.

$$
G(t)=e^{\frac{-\left(t-t_{0}\right)^{2}}{t_{h}^{2}}}, G(t+\Delta t)=b \cdot e^{\frac{-\left(t-t_{0}+\Delta t\right)^{2}}{\left(t_{h}+\Delta t_{h}\right)^{2}}}
$$

Here, $t_{0}$ is the time of the Gaussian pulse, $t_{h}$ is the $37 \%$ amplitude width of the Gaussian pulse, $b$ (slightly less than unity) defines the attenuation via a coefficient describing the decrease in amplitude of the pulse when propagating one time step and, $\Delta t_{h}$ is the increase in the $37 \%$ width of the pulse after propagating one time step. The transfer function related to the propagation of the pulse by one time-step is given by the ratio of the Fourier transforms (FFTs) of the above two signals.

$$
G(f)=\frac{F F T(G(t, 1))}{F F T(G(t, 0))}
$$

Here, $f$ represents the number of frequency components in the transfer function. The transfer function for the pulse to propagate two time steps is $G(f)^{2}$ and for $\mathrm{n}$ space steps, the transfer function will be $G(f)^{\mathrm{n}}$. Therefore, a matrix of transfer functions, $G(f, x)$, can be obtained that describes the propagation of the pulse from one node to another, across the sample thickness. This matrix is defined as:

$$
G(f, t)=\left[\begin{array}{ccccc}
1 & G(1) & G(1)^{2} & \cdots & G(1)^{n} \\
1 & G(2) & G(2)^{2} & \cdots & G(2)^{n} \\
1 & G(3) & G(3)^{2} & \cdots & G(3)^{n} \\
\vdots & \vdots & \vdots & \vdots & \vdots \\
1 & G(f) & G(f)^{2} & \cdots & G(f)^{n}
\end{array}\right]
$$

where $n$ represents the number of time steps, $\Delta \mathrm{t}$, required for the acoustic wave to traverse across the sample.

To obtain the output voltage waveform of the PEA system corrected for attenuation and dispersion in the frequency domain, the transfer function matrix, $G(f, t)$, was post multiplied with the row matrix of the simulated PEA output voltage, $V_{P V D F}(x)$. Here the $\mathrm{x}$-variable is replaced by the time, $t=x / c$, where $c$ is the propagation velocity of acoustic waves in the sample.

$$
V(f)=G(f, t) \cdot V_{P V D F}(t)
$$

\subsection{PVDF/BUFFER AMPLIFIER AND OSCILLOSCOPE FREQUENCY RESPONSE}

The PVDF/buffer amplifier and the oscilloscope frequency response work as a high pass filter (HPF) and low pass filter (LPF), respectively and lead to further distortion and imperfection in the PEA output signal [14]. The transfer function of the HPF can be represented by Equation (18):

$$
H(f)=\frac{j 2 \pi f R C}{1+j 2 \pi f R C}
$$

where, $R$ is the input resistance of the amplifier equal to $50 \Omega$. $C$ is the capacitance of the PVDF sensor given by $\left(\frac{\varepsilon_{0} \varepsilon_{r} A}{d}\right)$, where $\varepsilon_{0}=8.854 \times 10^{-12} \mathrm{~F} / \mathrm{m}$ is the vacuum permittivity; $\varepsilon_{r}$ is the relative permittivity of PVDF equal to 8.5 [10]; $A$ is the area of the PVDF sensor equal to $1 \mathrm{~cm}^{2}$, and $d$ is the PVDF thickness equal to $9 \mu \mathrm{m}$. Hence, the time constant of the $\mathrm{RC}$ circuit is $\sim 40 \mathrm{~ns}$. The transfer function of the LPF is given by:

$$
L(f)=\frac{1}{1+j \omega \tau_{r}}
$$

where, $\tau_{r}$ is the time constant of the low pass filter and is approximately equal to the reciprocal of the oscilloscope bandwidth $(500 \mathrm{MHz})$ and hence approximately $2 \mathrm{~ns}$.

The simulated PEA raw output signal corrected for attenuation/dispersion in the time domain, $V(t)$, requires multiplying the frequency domain representation of the output, $V(f)$, as calculated from equation (17), by the transfer function of these two filters, equations (18) and (19) and then taking the real part of the inverse FFT.

$$
V_{\text {raw }}(t)=\operatorname{Real}(\operatorname{IFFT}(V(f) \cdot L(f) \cdot H(f)))
$$

$V_{\text {raw }}(t)$ is the numerical result of the PEA simulation model which represents the PEA signal with the corresponding corrections for attenuation and dispersion, and for the buffer amplifier and oscilloscope responses. Perfect coupling of the acoustic pressure waves as they cross material interfaces was also assumed. This simulated voltage can then be compared directly with raw PEA experimental data provided wet coupling between the material interfaces is employed such as silicone oil used in this work. 


\subsection{MODEL PARAMETERS}

The model parameters used in the PEA simulations are given in Table 1.

Table 1. Model constants.

\begin{tabular}{|c|c|c|}
\hline Constant (unit) & Symbols & Values \\
\hline Spatial steps (m) & $d_{x}$ & $5 \times 10^{-7}$ \\
\hline Time steps (s) & $\Delta t$ & $10 \times 10^{-11}$ \\
\hline Gaussian pulse time delay (s) & $t_{p}$ & $5 \times 10^{-8}$ \\
\hline Width of the Gaussian pulse (s) & $t_{w}$ & $1.5 \times 10^{-9}$ \\
\hline Maximum simulation time (s) & $T_{\max }$ & $4 \times 10^{-7}$ \\
\hline
\end{tabular}

The thickness of each layer of the PEA apparatus and the corresponding material properties of each layer are summarized in Table $2[5,8,10,16]$. The initial values of the semicon top electrode were initially set to the values for the dielectric sample (LDPE) and the material parameters for the PMMA layer were set the same as that of the PVDF layer. This ensures, during the initial simulations of the ideal response of the PEA, there were no acoustic mismatch at the interfaces between the Semicon and the dielectric sample layers and at the interface between the PVDF and PMMA layers.

Table 2. Material properties used in the PEA simulation to produce ideal acoustic wave.

\begin{tabular}{|l|l|l|l|l|l|}
\hline Material & $\begin{array}{l}\text { Density } \\
\left(\mathrm{kg} / \mathrm{m}^{3}\right)\end{array}$ & $\begin{array}{l}\text { Velocity } \\
(\mathrm{m} / \mathrm{s})\end{array}$ & $\begin{array}{l}\text { Effective } \\
\text { modulus } \\
(\mathrm{GPa})\end{array}$ & $\begin{array}{l}\text { Thickness } \\
(\mathrm{mm})\end{array}$ & $\begin{array}{l}\text { Relative } \\
\text { permitti } \\
\text { vity }\end{array}$ \\
\hline $\begin{array}{l}\text { Top } \\
\text { absorber }\end{array}$ & 2700 & 6295 & 107 & 5 & - \\
\hline Top Al & 2700 & 6295 & 107 & 5 & - \\
\hline Semicon & 917 & 2085 & 3.987 & 1 & - \\
\hline Sample & 917 & 2085 & 3.987 & 0.15 & 2.3 \\
\hline $\begin{array}{l}\text { Bottom } \\
\text { Al }\end{array}$ & 2700 & 6295 & 107 & 10 & - \\
\hline PVDF & 1200 & 2692 & 8.7 & 0.009 & 8.5 \\
\hline PMMA & 1200 & 2692 & 8.7 & 5 & 4 \\
\hline $\begin{array}{l}\text { Bottom } \\
\text { absorber }\end{array}$ & 1200 & 2692 & 8.7 & 5 & - \\
\hline
\end{tabular}

\section{EXPERIMENTAL}

In parallel with the simulations, experimental PEA measurements were carried out on $150 \mu \mathrm{m}$ thickness thin films of cross-linked polyethylene (XLPE) samples. The samples were manufactured using the procedure described in [17]. The samples were degassed in a vacuum oven at $60^{\circ} \mathrm{C}$ for $24 \mathrm{~h}$ prior to testing to remove volatile impurities. When clamping the sample in the PEA system silicone oil was used to aid acoustic coupling at the sample-electrode interfaces.

The amplitude of the applied voltage pulses was $500 \mathrm{~V}$ and the pulse width was $8 \mathrm{~ns}$. A $1 \mathrm{kV}$ DC voltage was applied to the sample and the PEA signal was recorded for $10 \mathrm{~s}$ after the voltage application. The measurements were carried out at room temperature. At such a low electric field and short time duration, space charge should not be accumulated in the sample. This was verified by longer term space charge measurements. In order to investigate the effect of the material properties of the top electrode material on the PEA output signal, experiments were performed with two top electrode configurations: 1) Semicon was used as the top electrode and the bottom electrode was made out of aluminum; 2) both the top and bottom electrodes were made out of aluminum.

\section{Results \\ 4.1 PEA EXPERIMENTAL RESULTS}

Figure 2 shows raw PEA measurement data without calibration and correction as described in section 3 . The charge at the bottom $\mathrm{Al}$ electrode/sample interface is represented in Figure 2 by a negative voltage peak followed by a small positive peak (overshoot). The voltage steadily decreases from this positive overshoot peak with time until the positive peak representing the electrode charge at the top electrode/sample interface. In the absence of space charge, the voltage peaks at both electrodes should be (in principle) equal in magnitude and opposite in polarity. However, in the example shown here, the positive peak has a lower magnitude and a larger width (duration) than the negative peak.

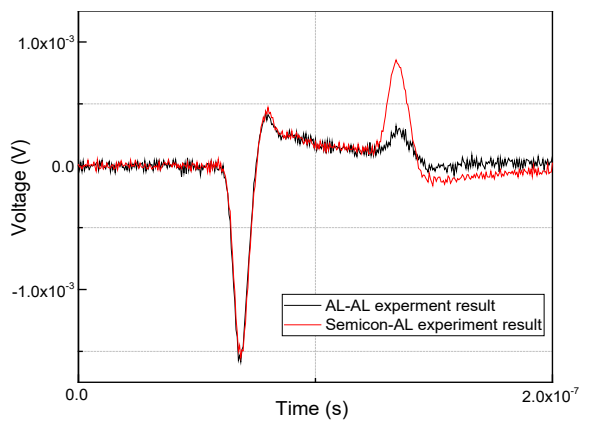

Figure 2. Raw experimental PEA measurement results. Red- signal obtained with Semicon top electrode. Black- After semicon replaced by aluminium.

Comparing the measurement results obtained with Semicon as the top electrode before and after replacing with Aluminium, demonstrated that the amplitude and duration of the first peak as well as the overshoot and voltage decay between the peaks remained unchanged. However, in common with previous work [18] the amplitude of the second peak was reduced significantly when aluminium was used as the top electrode and was attributed to the acoustic mismatch introduced between the sample and aluminium when used as the top electrode. See Bernstein [14] where this is discussed in more detail.

\subsection{PEA SIMULATION RESULTS}

Simulations of 1D acoustic wave propagation were carried out for a number of case studies that are described below. In all simulations, the sample was assumed to be free of space charge, $\rho(\mathrm{x}, \mathrm{t})=0$, and therefore the electric field, $\mathrm{E}(\mathrm{x}, \mathrm{t})$ in a thin film sample can be assumed to be distributed uniformly. The temperature was assumed to be isothermal at $20{ }^{\circ} \mathrm{C}$ and all material parameters are assumed constant over their thickness.

\subsubsection{INITIAL PEA SIMULATIONS}

The acoustic properties of the semicon and polymer sample, and the acoustic properties of PVDF and PMMA were initially assumed to be the same therefore removing the possibility of acoustic reflections occurring at these interfaces. For these initial simulations, corrections for attenuation and dispersion and the PVDF/amplifier/oscilloscope were not carried out. The simulation therefore relates to an ideal case in which no signal distortions should occur. 
The positions of the pressure waves within the PEA apparatus at various times of propagation are shown in Figure 3. Figure $3 \mathrm{a}$ shows the acoustic pressure wave for time, $\mathrm{t}=0$, across the eight subdomains of the PEA model as produced by the MATLAB code. The sample/bottom Al electrode interface is designated ' $A$ ' and that of the semicon/sample interface, 'B'. At time $\mathrm{t}=0$, the pulsed voltage had not yet been applied and the pressure waves had not yet been generated. After the pulse delay time, $t_{p}$, compressive (positive) pressure is generated within the sample due to the applied pulse voltage as shown in Figure $3 b$. Here, the sample is under uniform compression. After the voltage pulse had receded, the pressure within the sample bulk returns to zero as shown in Figure 3c, but pressure waves that were generated at the two electrode/insulator interfaces, A and B propagate in the direction shown by the arrows. In fact, two waves propagate from each source (interface), a compressive wave in one direction and a rarefaction wave in the opposite direction. The pressure waves that propagate towards and through the bottom $\mathrm{Al}$ electrode and to the PVDF sensor form the useful signal. These are identified as pulse ' 1 ' from interface A and pulse ' 2 ' from interface B, while the other waves moved towards the top $\mathrm{Al}$ electrode and away from the PVDF detector. Figure $3 \mathrm{~d}$ shows the pressure waves at the time when the acoustic pulse generated at interface A had propagated one third of the way through the bottom $\mathrm{Al}$ electrode. In Figure 3e the same pressure wave (pulse 1) has approached the PVDF detector layer. Part of this acoustic pulse propagates through the PVDF sensor whilst the reflected pressure wave propagates back towards the sample. At the same time, pulse 2 had reached interface A where this is partly transmitted and reflected. In Figure 3f, the pulse 1 has propagated into the PMMA layer whilst pulse 2 approaches the sample/PVDF interface. Figure $3 \mathrm{~g}$ shows the pressure pulse 1 being attenuated in the PML layer and pulse 2 propagating in the PMMA layer having passed through the PVDF layer. At each simulation time step, the pressure wave across the PVDF thickness was integrated over the thickness of the PVDF layer to give the time dependent PVDF output voltage, $V_{\text {out }}(t)$. A typical idealised PEA output waveform, $\mathrm{V}_{\text {out }}(\mathrm{t})$, is shown in Figure 4. In Figure 4, two voltage pulses are shown to occur sequentially in time. The first is due to the pressure pulse 1 produced at the A interface followed by an opposite polarity voltage pulse due to the pressure pulse 2 that was produced at interface B. The two peaks have approximately equal amplitudes and represent the surface electrode charges that are responsible for establishing the internal electric field in the sample.

\subsubsection{PEA LINEARITY AND PULSE POLARITY}

The source term for the acoustic pressure waves, as given in Equation (5), consists of two terms. The first is linear whilst the second term is quadratic with respect to the pulsed field, $\Delta E$, giving rise to a non-linear response when $E \leq \Delta E$. Secondly, the amplitude of the acoustic waves will be dependent on the relative sign of the pulse field, $\Delta E$, compared to that of the DC applied field, E. In order to show the effect of the pulse voltage on the PEA output signal, a series of simulations were performed at a fixed value of pulse voltage $\Delta V=500 \mathrm{~V}$ and varying applied DC voltage between -2 to $2 \mathrm{kV}$.

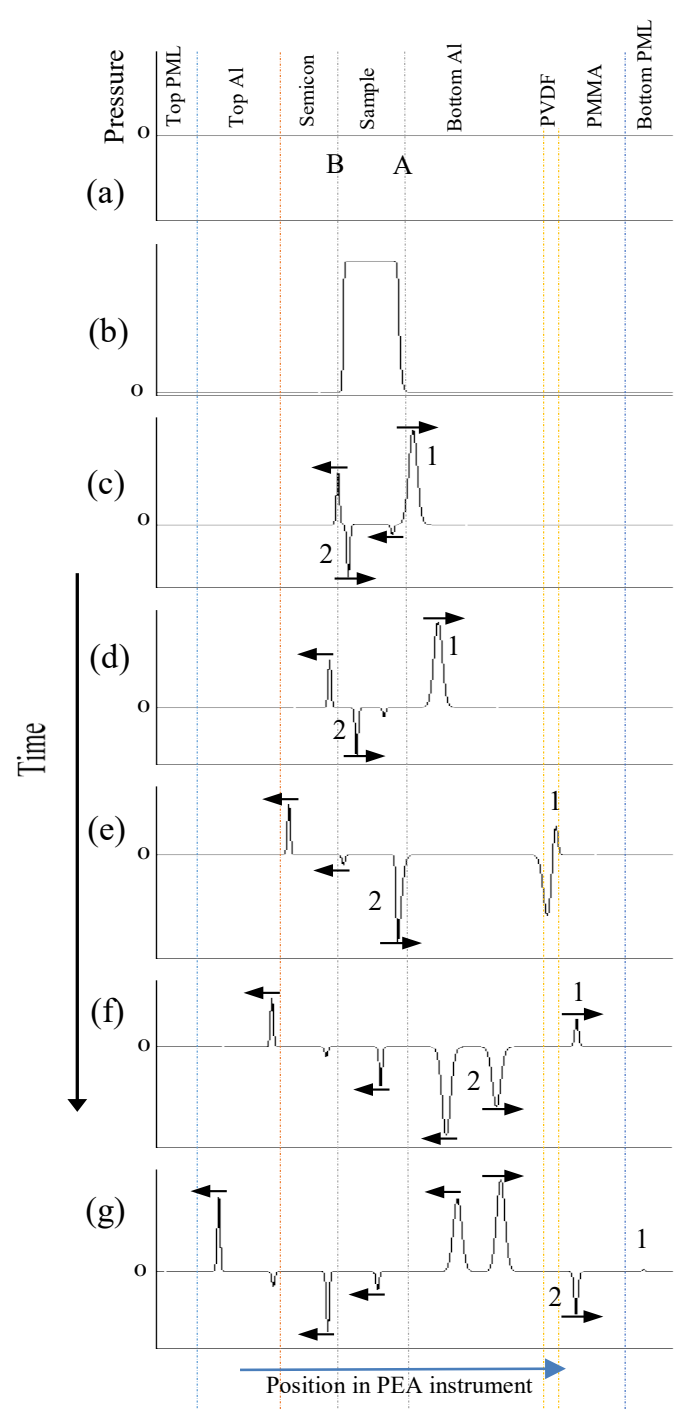

Figure 3. (a)-(g) Pressure wave propagating through the PEA layers at various times. The direction of propagation is shown by the arrows.

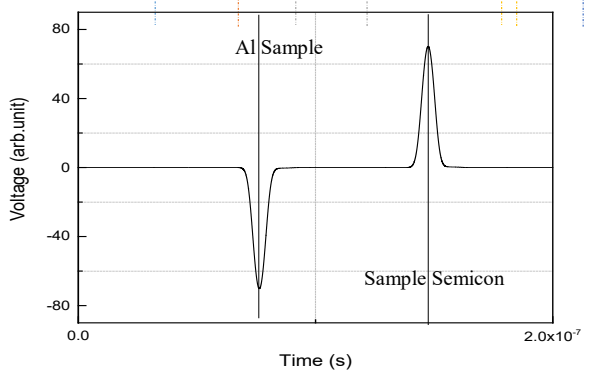

Figure 4. Output voltage from the PVDF sensor in case of an ideal (lossless) sample with matching acoustic layers. Note the waveform has been inverted to take into account signal inversion in the sensor/amplifier for direct comparison with the experimental raw PEA data shown in figure 2.

The results of these simulations are shown in figure 5. The results demonstrate that if the polarity of the applied DC voltage was the same as the pulse voltage (positive), the interface electrode peaks were significantly higher in comparison with the instances where the polarity of the applied DC voltage was negative. To examine further the linearity of the PEA response, these simulations were extended to a wider range of positive and negative applied DC voltages. 


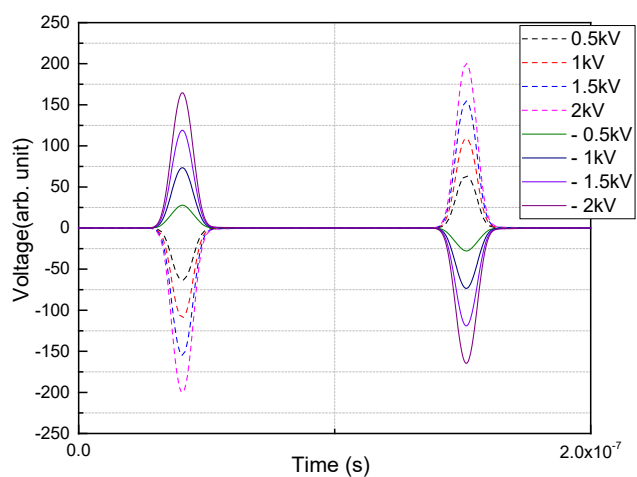

Figure 5. PEA output signal, $500 \mathrm{~V}$ pulse for different DC applied voltages.
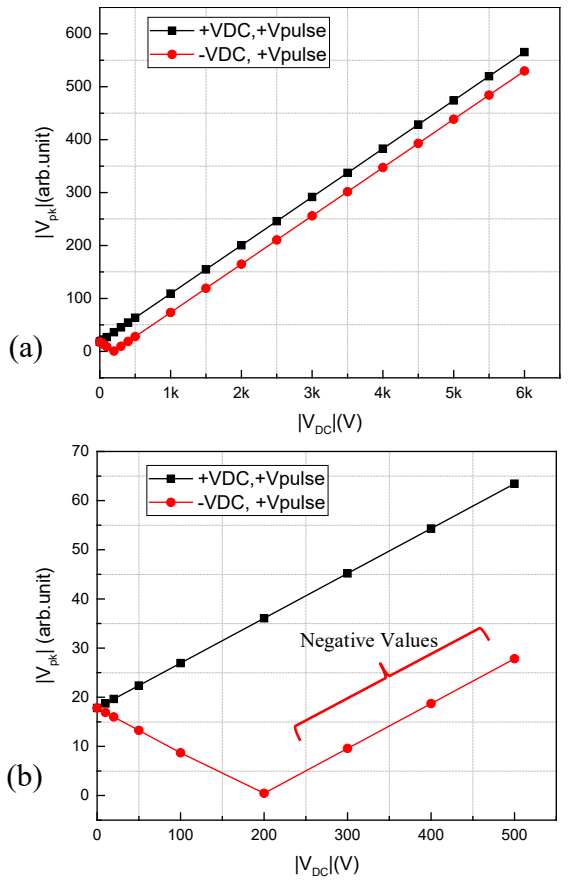

Figure 6. Magnitude of the simulated PEA aluminium/sample interface peak in an ideal (lossless) sample against magnitude of applied DC voltage. Pulse voltage $500 \mathrm{~V}$, (a) DC applied voltages from -6 to $+6 \mathrm{kV}$. (b) DC applied voltages from -500 to $+500 \mathrm{~V}$.

In Figure 6, the magnitude of the aluminium/sample interface peak was plotted against the magnitude of the positive and negative applied DC voltage. For voltages greater than $200 \mathrm{~V}$, the response of the PEA is linear except for an offset depending on whether the pulsed field acted with or against the applied DC voltage field. This offset can be allowed for by performing separate PEA calibration for the two polarities of applied voltage. The voltage for the crossover between a positive and negative peak was found to be different from that anticipated theoretically from Equation (5), where the source term, can be written, $\Delta M \propto V_{D C} \Delta V+\left(\frac{1}{2}\right) \Delta V^{2}$. For the source term to be zero, the two terms on the right hand side must cancel. For a positive pulse voltage, $\Delta V=500 \mathrm{~V}$, as used in the simulation, the two terms should cancel when the applied voltage, $V_{D C}$ is equal to $-250 \mathrm{~V}$. However in the simulation, the pulse voltage, takes the form of a Gaussian pulse and simulations demonstrate that at the instant of time at the peak of the Gaussian pulse (500 V) the balancing of the two terms occurs as predicted by theory. However during the rising and falling edges of the Gaussian pulse, the balancing of the two terms in equation 5 does not hold and in this case two acoustic pressure waves are generated, one during the rising edge of the pulse voltage and the other during the falling edge of the pulse voltage. These two pressure waves are integrated over the thickness of the PVDF sensor to give a finite PEA output voltage pulse. To get a near zero PEA response, it is necessary to balance the acoustic pressure wave pulses produced on the rising and falling edges of the pulse voltage with an opposite pulse produced by altering the applied DC voltage such that an opposite polarity pulse is produced of sufficient magnitude to achieve cancelling of the integrated pressure waves over the thickness of the PVDF sensor. In the simulations this condition is met when, $V_{D C}=-200 \mathrm{~V}$. In fact perfect cancelling of these two contributions, that due to the rising and falling edges of the applied pulse voltage and that due to the applied DC voltage, cannot occur precisely due to their different timings.

\subsubsection{PEA SYSTEM WITH MISMATCHING ACOUSTIC LAYERS AND A LOSSLESS SAMPLE}

So far, the material properties of PMMA and PVDF layers were kept the same in these initial simulations in order to eliminate acoustic wave reflections at the PVDF/PMMA interface. Figure 7 shows the idealised PEA simulated output voltages when the value of effective modulus of PMMA was varied between 6.7 and $10.7 \mathrm{GPa}$ whilst keeping all other material properties to the values given in Table 2 . When the effective modulus of the PMMA layer was equal to $8.7 \mathrm{GPa}$ (i.e. the same as PVDF), no reflection adjacent the electrode peaks was observed. However when different values of effective modulus of the PMMA layer were used, acoustic reflections occurred (shown in Figure 7 adjacent to the electrode peaks), with the sign of the peak distortion depending upon whether the modulus of PMMA was greater (positive) or smaller (negative) than that of PVDF. However the peak values of the two electrode interfaces were unaffected. Changing the PMMA parameters to those known from the literature [19], density, $1760 \mathrm{~kg} / \mathrm{m}^{3}$, and effective modulus of PVDF, $10.8 \mathrm{GPa}$, in the simulation yielded the PEA simulated waveform shown in Figure 8.

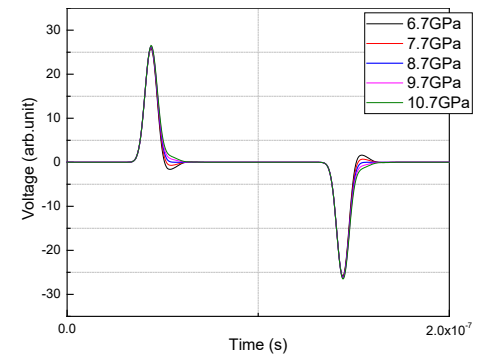

Figure 7. Simulation of PEA output in case where the modulus of PVDF is fixed at $8.7 \mathrm{GPa}$ and the values of the PMMA effective modulus were varied.

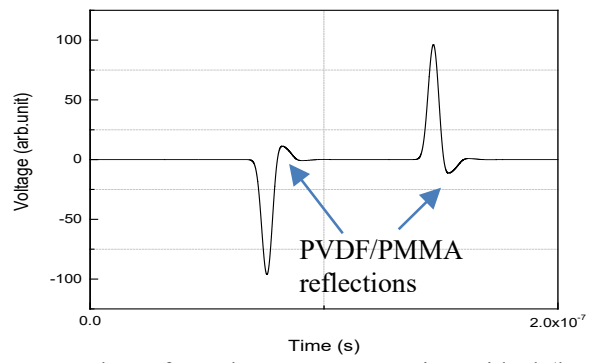

Figure 8. Output voltage from the PVDF sensor in an ideal (lossless) sample with acoustic reflection between PVDF and PMMA layers. 
Figure 8 demonstrates that acoustic reflections at the PVDF/PMMA interface will cause an overshoot in the electrode charge peaks. In this case, part of the acoustic wave was reflected at the PVDF-PMMA interface back through the PVDF sensor and the reflected wave interfered with the incident wave. These unwanted reflections can potentially lead to misinterpretation of the PEA response in terms of space charge regions that actually do not exist. When applying deconvolution techniques to remove system artefacts it is necessary to ensure a space charge free calibration measurement. This is difficult to assess when overshoots are present in the raw PEA data.

Simulations were also performed in the case of varying effective modulus of the semicon layer from $2 \mathrm{GPa}$ to $6 \mathrm{GPa}$ whilst all other material parameters were kept at the values given in Table 2. Figure 9 shows the resultant simulations of the idealised PEA signal. At 4 GPa both the semicon electrode and the dielectric material have the same value of the effective modulus. However decreasing or increasing the value of the effective modulus of the semicon relative to that of the insulator leads to a change in the amplitude of the second peak corresponding to the position of the semicon/insulator interface whilst the peak corresponding to the aluminium electrode is unchanged. Such an effect could potentially be confused with the phenomena of attenuation of the acoustic pressure waves as they pass through the sample (see next section) and could potentially be significant when sample materials having different acoustic properties than semicon are to be tested.

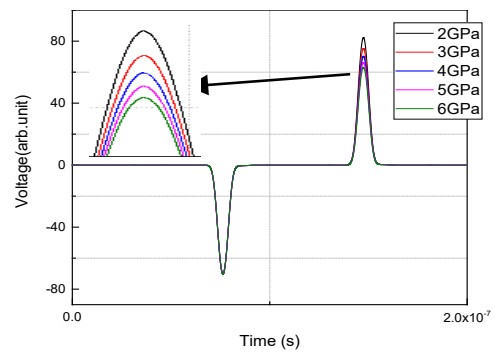

Figure 9. Effect of electrode properties on the PEA output signal.

\subsubsection{EFFECT OF ATTENUATION AND DISPERSION}

The data processing techniques of section 2.3 were employed to correct the idealised PEA response for attenuation and dispersion of the acoustic waves as they traverse the sample. Figure 10 shows the effect of attenuation and dispersion on PEA output signal using a range of attenuation coefficients ( $b$ from 0.97 to 0.99$)$ and dispersion coefficients $\left(\Delta t_{h}=1.25\right.$ to 1.85 ). The attenuation and dispersion only affect the second peak (Semicon/sample interface), as shown in Figure 10 as the signal from the aluminium/sample interface does not pass through the sample. As a consequence, no changes were observed to the aluminium/sample interface peak but absorption decreases and dispersion widens the second peak associated with the sample/semicon interface. It should be pointed out that attenuation and dispersion have been treated separately in the analysis for simplicity and convenience rather than strict adherence to their Kramers-Kronig interrelationship.

\subsubsection{PVDF/AMPLIFIER FREQUENCY RESPONSE}

As pointed out in section 2.4, the electrical circuit formed by the PVDF capacitance and buffer amplifier input resistance works as a high pass filter whilst the oscilloscope bandwidth acts as a low pass filter. In order to simulate the raw PEA output signal it is necessary to take these factors into account.

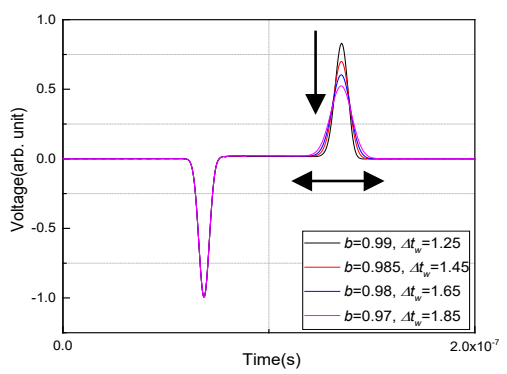

Figure 10. Effect of attenuation and dispersion on the PEA output signal.

The value of buffer amplifier input resistance employed in the experimental set up was $50 \Omega$. PEA simulations were performed over a range of input resistance values, both above and below $50 \Omega$, to show the effect of the PVDF/buffer amplifier response on the PEA output signal whilst keeping the oscilloscope bandwidth constant at $500 \mathrm{MHz}$. The parameter values given in Table 2 were used in the PEA simulation and acoustic attenuation and dispersion was also included following the procedure given in Section 2.3. Values of attenuation coefficient, $b=0.965$ and dispersion coefficient, $\Delta t_{h}=1.8$ were chosen in order to match approximately the raw PEA experimental data presented in Figure 2. The results of these simulations are shown in Figure 11. In all cases the high pass filter response leads to an overshoot in the voltage following each peak followed by an exponential decay. The degree of overshoot and the rate of decay become greater with decreasing input resistance.

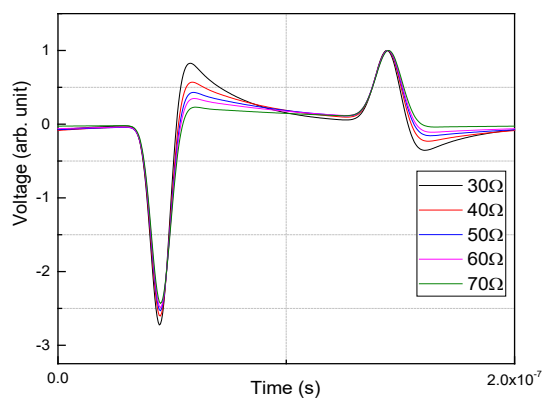

Figure 11. PEA output at different values of amplifier input resistance.

\subsubsection{COMBINED PEA SIMULATIONS}

Combined PEA simulations including acoustic reflections, attenuation and dispersion correction and the $\mathrm{PVDF} /$ amplifier/oscilloscope response were conducted in order to reproduce the experimental data of Figure 2. The simulation parameters of Table 2 were used along with the actual values of the material parameters for PMMA as given in Section 4.2.3, the attenuation and dispersion parameters from Section 4.2.5 and an amplifier input resistance of $50 \Omega$. The simulations were performed for two cases, firstly with the semicon material as the top electrode and then after substituting the material properties of aluminium for the semicon layer. The results of the simulations along with the experimental data are shown in Figure 12. The PEA simulations were successful in reproducing all the essential features of the experimental PEA response. 


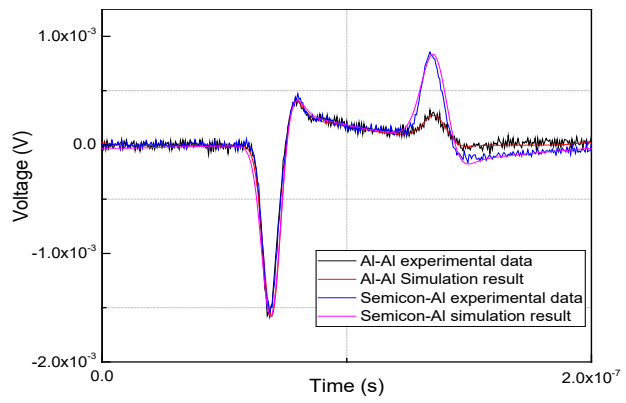

Figure 12. Effect of the top electrode material on the raw PEA output data.

\section{Discussion}

The development of the PEA simulation model is essentially based on the physics of body forces in dielectrics [9] coupled with the theory of acoustic wave propagation in elastic media. The model has been further developed to include acoustic reflections at the various interfaces of the PEA instrument, the attenuation and dispersion of acoustic pressure waves and the instrumental response of the sensor driving the $50 \Omega$ input impedance amplifier. The overall instrumental response can therefore be predetermined from a given PEA experimental configuration. The PEA simulation model, as it is described here, applies to the case of space charge free material and can be used to provide baselines for comparison with experimentally derived calibration data. Furthermore, electric field distributions predicted from charge transport theory can be used as the input to the PEA simulation to predict raw PEA data that can be compared directly to experimental PEA data. The PEA simulation model therefore provides a direct link between charge transport theory and experiment without the need of employing unstable numerical techniques to recover the space charge profile. Deficiencies of the deconvolution technique include: (1) to correct for the high pass sensor/amplifier response the deconvolution boosts the low frequency components including noise. This causes false detail to appear in the resultant PEA output signal and can shift the baseline voltage between the interface peaks and yield significant errors in the calculated electric field distribution. (2) Taking the inverse of an ill-conditioned $\mathrm{G}$ matrix can result in significant false detail to appear in the corrected PEA signal. This can potentially arise if there are small timing shifts between the interface charges due to a slight change of sample temperature.

\section{Conclusions}

The numerical model proposed in this work offers an alternative numerical procedure for the calibration and interpretation of PEA measurement signals without employing a deconvolution algorithm. Hence, the model allows the relative contribution of the various factors affecting the PEA measurements to be assessed and quantified, which otherwise if unaccounted for, could contribute to systematic errors in the measured space charge profiles. This allows the space charge profiles to be obtained directly from the measured signal without any prior assumptions.

\section{REFERENCES}

[1] Y. Li and T. Takada, "Progress in space charge measurement of solid insulating materials in Japan," IEEE Electr. Insul. Mag., vol. 5, pp. 1628, 1994.

[2] Y. Li, M. Yasuda, and T. Takada, "Pulsed electroacoustic method for measurement of charge accumulation in solid dielectrics," IEEE Trans. Dielectr. Electr. Insul., vol. 1, pp. 188-195, 1994.

[3] T. Maeno et al, "Measurement of spatial charge distribution in thick dielectrics using the pulsed electroacoustic method," IEEE Trans. Dielectr. Electr. Insul., vol. 23, pp. 433-439, 1988.

[4] S. Hole, "Behind space charge distribution measurements," IEEE Trans. Dielectr. Electr. Insul., vol. 4, pp. 1208-1214, 2012.

[5] M. Wadamori et al, "Proposal of numerical analysis model of acoustic wave propagation and generation on PEA method," IEEE Int. Conf. Prof. Appl. Dielectr. Mat. (ICPDM), 2003, pp. 863-866.

[6] G. Chen, Y. Chong, and M. Fu, "Calibration of the pulsed electroacoustic technique in the presence of trapped charge," Measurement Science and Technology, vol. 17, p. 1974, 2006.

[7] Y. Li, M. Aihara, K. Murata, Y. Tanaka, and T. Takada, "Space charge measurement in thick dielectric materials by pulsed electroacoustic method," Rev. Scientific Instruments, vol. 66, pp. 3909-3916, 1995.

[8] H. Zheng, "Application of PEA technique to space charge measurement in cylindrical geometry HV cable systems," $\mathrm{PhD}$ dissertation, University of Leicester, Department of Engineering, 2015.

[9] S. Hole, T. Ditchi, and J. Lewiner, "Non-destructive methods for space charge distribution measurements: what are the differences?," IEEE Trans. Dielectr. Electr. Insul., vol. 10, pp. 670-677, 2003.

[10] R. Bodega, P. Morshuis, and J. Smit, "Space charge measurements on multi-dielectrics by means of the pulsed electroacoustic method," IEEE Trans. Dielectr. Electr. Insul., vol. 13, pp. 272-281, 2006.

[11] S.Hole, V. Griseri, L. A. Dissado, and J. C. Fothergill, "Improvement of PEA signal analysis using simulations for complex geometry samples," J. Phys. D: Appl. Phys., vol. 35, p. 19-24, 2001.

[12] P. M. Morse and K. U. Ingard, Theoretical Acoustics, Princeton university press, 1968

[13] Y. Xiaojuen, D. Borup, J. W. Wiskin, M. Berggren, R. Eidens, and S. A. Johnson, "Formulation and validation of Berenger's PML absorbing boundary for the FDTD simulation of acoustic scattering," IEEE Trans. Ultrasonics, Ferroelectrics and Frequency Control, vol. 44, pp. 816-822, 1997.

[14] J. B. Bernstein, "Analysis of the electrically stimulated acoustic-wave method for observing space charge in semi-insulating films," Physical Review B, Vol. 44, No., 19, pp10804-14, 1991.

[15] Y. Tanaka, K. Hanawa, K. Suzuki, and T. Takada, "Attenuation recovery technique for acoustic wave propagation in PEA method," Annu Rep. Conf. Electr. Insul. Dielectr. Phenom. (CEIDP), 2001, pp. 407-410.

[16] G. Kaye, Kaye \& Laby-Tables of Physical and Chemical Constants, Longman, 1995.

[17] W. Lei, K et al, "Are nano-composites really better DC insulators? A study using silica nanoparticles in XLPE," IEEE Trans. Dielectr. Electr. Insul., vol. 24, pp. 2268-2270, 2017.

[18] K. Fukunaga, "Progress and prospects in PEA space charge measurement techniques," IEEE Electr. Insul. Mag., vol. 24, pp. 26-37, 2008.

[19] M. A. A. L. Arnaout, Acoustic Wave Propagation in a Pulsed Electro Acoustic Cell, INTECH Open Access Publisher, 2013.

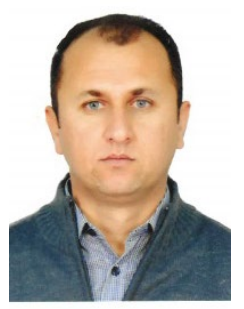

Awat A. Mulla was born in Qalladze, Sulaimani, Iraq, in 1983. He graduated from the Salahaddin UniversityErbil, Iraq in 2005 with a BSc degree in Electrical Engineering/Power and Machine and gained MSc degree in Electrical Engineering/Power and Machine from Sulaimani University, Iraq, in 2012. He joined the High voltage group at Leicester University, UK in 2015 to study for a $\mathrm{PhD}$. He was awarded a $\mathrm{PhD}$ degree in 2019. His main research interest is modelling and measuring the electrical properties and space charge of polymer insulating materials in HVDC applications. 